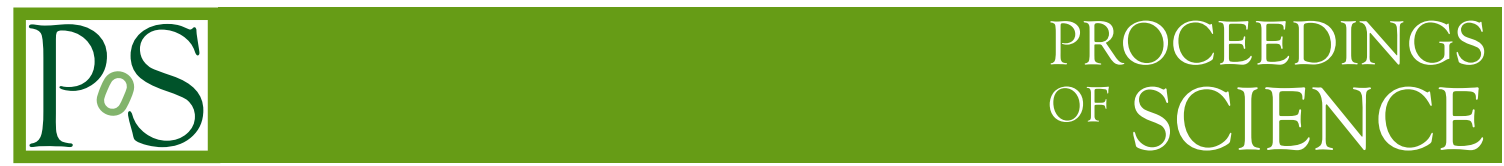

MBR Monte Carlo Simulation in PYTHIA8

\title{
Robert Ciesielski*
}

The Rockefeller University, 1230 York Avenue, New York, NY 10065, USA

E-mail: robert.ciesielski@rockefeller.edu

\section{Konstantin Goulianos}

The Rockefeller University, 1230 York Avenue, New York, NY 10065, USA

E-mail: dino@rockefeller.edu

\begin{abstract}
We present the MBR (Minimum Bias Rockefeller) Monte Carlo simulation of (anti)proton-proton interactions and its implementation in the PYTHIA8 event generator. We discuss the total, elastic, and total-inelastic cross sections, and three contributions from diffraction dissociation processes that contribute to the latter: single diffraction, double diffraction, and central diffraction or double-Pomeron exchange. The event generation follows a renormalized-Regge-theory model, successfully tested using CDF data. Based on the MBR-enhanced PYTHIA8 simulation, we present cross-section predictions for the LHC and beyond, up to collision energies of $50 \mathrm{TeV}$.
\end{abstract}

36th International Conference on High Energy Physics,

July 4-11, 2012

Melbourne, Australia

${ }^{*}$ Speaker. 


\section{Introduction}

The data from the LHC have shown that there exists no Monte Carlo simulation which is able to satisfactory predict all the features of the so-called soft physics processes at high energies. An important component in the soft domain are diffractive interactions, which constitute a significant fraction of the total inelastic proton-proton cross section.

The MBR (Minimum Bias Rockefeller) Monte Carlo (MC) simulation[1] is an event generator which predicts the energy dependence of the total, elastic, and total-inelastic $p p$ cross sections, and fully simulates the main diffractive components of the total-inelastic cross section: single dissociation (SD), double dissociation (DD), and central dissociation (CD) or double-Pomeron exchange (DPE):

$$
\begin{array}{r}
\text { SD } \quad p p \rightarrow X p \\
\text { or } \quad p p \rightarrow p Y \\
\text { DD } \quad p p \rightarrow X Y \\
\mathrm{CD}(\mathrm{DPE}) \quad p p \rightarrow p X p
\end{array}
$$

where $X$ and $Y$ represent diffractively dissociated protons. The diffractive-event generation in MBR is based on a phenomenological renormalized-Regge-theory model[2], which is unitarized by interpreting the Pomeron flux as the probability for forming a diffractive rapidity gap.

The MBR model was originally developed for the CDF experiment at the Tevatron, and successfully tested with CDF diffractive results. It has been recently implemented in PYTHIA 8.165[3], where it can be activated with the flag Diffraction: $\mathrm{PomFlux}=5$.

In this paper, we briefly summarize the MBR simulation and its implementation in PYTHIA8. More details can be found in Ref. [1].

\section{The MBR model}

In the MBR model the $\sigma_{\mathrm{tot}}^{p^{ \pm} p}(s)$ cross sections at a $p p$ center-of-mass-energy $\sqrt{s}$ are calculated as follows:

$$
\begin{array}{ll}
\sigma_{\mathrm{tot}}^{p^{ \pm} p}=16.79 s^{0.104}+60.81 s^{-0.32} \mp 31.68 s^{-0.54} & \text { for } \sqrt{s}<1.8 \mathrm{TeV}, \\
\sigma_{\mathrm{tot}}^{p^{ \pm} p}=\sigma_{\mathrm{tot}}^{\mathrm{CDF}}+\frac{\pi}{s_{0}}\left[\left(\ln \frac{s}{s_{F}}\right)^{2}-\left(\ln \frac{s^{\mathrm{CDF}}}{s_{F}}\right)^{2}\right] & \text { for } \sqrt{s} \geq 1.8 \mathrm{TeV},
\end{array}
$$

where the term for $\sqrt{s}<1.8 \mathrm{TeV}$ is obtained from a global Regge-theory fit to pre-LHC data on $p^{ \pm} p, K^{ \pm} p$ and $\pi^{ \pm} p$ cross sections [4], while that for $\sqrt{s} \geq 1.8 \mathrm{TeV}$ is a prediction of a model based on a saturated Froissart bound[5]. The latter is normalized to the CDF measurement of $\sigma_{\text {tot }}$ at $\sqrt{s^{\mathrm{CDF}}}=1.8 \mathrm{TeV}, \sigma_{\mathrm{tot}}^{\mathrm{CDF}}=80.03 \pm 2.24 \mathrm{mb}$, and depends on two parameters: the energy at which the saturation occurs, $\sqrt{s_{F}}=22 \mathrm{GeV}$, and an energy-scale parameter, $s_{0}=3.7 \pm 1.5 \mathrm{mb}$.

The elastic cross section, $\sigma_{\mathrm{el}}^{p^{ \pm}} p$, is calculated using $\sigma_{\mathrm{tot}}$ from the above equations multiplied by the elastic-to-total cross-section ratio, $\sigma_{\mathrm{el}} / \sigma_{\mathrm{tot}}$, obtained from the global Regge fit of Ref. [4]. The total inelastic cross section is calculated as $\sigma_{\text {inel }}=\sigma_{\text {tot }}-\sigma_{\mathrm{el}}$. 
Diffractive SD, DD and CD (or DPE) cross sections are calculated using a phenomenological model discussed in detail in Ref. [2]. Differential cross sections are expressed in terms of the Pomeron $(\mathbb{P})$ trajectory, $\alpha(t)=1+\varepsilon+\alpha^{\prime} t=1.104+0.25 \mathrm{GeV}^{-2} \cdot t$, the Pomeron-proton coupling, $\beta(t)$, and the ratio of the triple- $\mathbb{P}$ to the $\mathbb{P}$-proton couplings, $\kappa \equiv g(t) / \beta(0)$. For sufficiently large rapidity gaps $(\Delta y \gtrsim 3)$, for which $\mathbb{P}$-exchange dominates, the cross sections may be written as,

$$
\begin{aligned}
\frac{d^{2} \sigma_{S D}}{d t d \Delta y} & =\frac{1}{N_{\text {gap }}(s)}\left[\frac{\beta^{2}(t)}{16 \pi} e^{2[\alpha(t)-1] \Delta y}\right] \cdot\left\{\kappa \beta^{2}(0)\left(\frac{s^{\prime}}{s_{0}}\right)^{\varepsilon}\right\}, \\
\frac{d^{3} \sigma_{D D}}{d t d \Delta y d y_{0}} & =\frac{1}{N_{\text {gap }}(s)}\left[\frac{\kappa \beta^{2}(0)}{16 \pi} e^{2[\alpha(t)-1] \Delta y}\right] \cdot\left\{\kappa \beta^{2}(0)\left(\frac{s^{\prime}}{s_{0}}\right)^{\varepsilon}\right\}, \\
\frac{d^{4} \sigma_{D P E}}{d t_{1} d t_{2} d \Delta y d y_{c}} & =\frac{1}{N_{\text {gap }}(s)}\left[\Pi_{i}\left[\frac{\beta^{2}\left(t_{i}\right)}{16 \pi} e^{2\left[\alpha\left(t_{i}\right)-1\right] \Delta y_{i}}\right]\right] \cdot \kappa\left\{\kappa \beta^{2}(0)\left(\frac{s^{\prime}}{s_{0}}\right)^{\varepsilon}\right\},
\end{aligned}
$$

where $t$ is the square of the four-momentum-transfer at the proton vertex and $\Delta y$ is the rapidity gap width. The variable $y_{0}$ in Eq. (2.4) is the center of the rapidity gap. In Eq. (2.5), the subscript $i=1,2$ enumerates Pomerons in the DPE event, $\Delta y=\Delta y_{1}+\Delta y_{2}$ is the total (sum of two gaps) rapidity-gap width in the event, and $y_{c}$ is the center in $\eta$ of the centrally-produced hadronic system.

Eqs. (2.3) and (2.4), up to a renormalization parameter $N_{\text {gap }}(s)$, are equivalent to those of standard-Regge theory, as $\xi$, the fractional forward-momentum-loss of the surviving proton (forward momentum carried by $\mathbb{I P}$ ), is related to the rapidity gap by $\xi=e^{-\Delta y}$. The variable $\xi$ is defined as $\xi_{\mathrm{SD}}=M^{2} / s$ and $\xi_{\mathrm{DD}}=M_{1}^{2} M_{2}^{2} /\left(s \cdot s_{0}\right)$, where $M\left(M_{1}, M_{2}\right)$ are the masses of dissociated systems in SD (DD) events. For DD events, $y_{0}=\frac{1}{2} \ln \left(M_{2}^{2} / M_{1}^{2}\right)$, and for DPE $\xi=\xi_{1} \xi_{2}=M^{2} / s$.

The renormalization parameter, $N_{\text {gap }}(s)$, is defined as $N_{\text {gap }}(s)=\min (1, f)$, where $f$ is the integral of the term in square brackets, corresponding to a "Pomeron flux". The integral is calculated over all phase space in $t_{i}$ and in $\eta_{0}$ (DD) or $\eta_{c}$ (DPE) for $\Delta y>2.3$. The renormalization procedure, based on interpreting the Pomeron flux as a (diffractive) gap-formation probability, yields predictions which are in very good agreement with diffractive measurements at $\operatorname{CDF}[6,7,8]$.

\section{Comparison with experimental results}

In Fig. 3, the predictions of the MBR model are compared to the measurements of the total, total-inelastic, the elastic $p p$ cross sections at $\sqrt{s}=7 \mathrm{TeV}$ performed by the TOTEM experiment[9], showing excellent agreement between the data and the model. Figure 2 shows a comparison between the MBR calculations for the single-diffractive and double-diffractive cross sections and the measurements of the ALICE experiment at $\sqrt{s}=0.9,2.76$ and $7 \mathrm{TeV}[10]$. The MBR predictions are also in good agreement with the diffractive data.

\section{Implementation in PYTHIA8}

The MBR generation has been made available in PYTHIA8, starting from version PYTHIA8.165, where it can be activated with the flag Diffraction:PomFlux $=5$. The simulation is valid for $p p, p \bar{p}$ and $\bar{p} p$ scattering. The CD (DPE) process is implemented in PYTHIA 8 for the first time. It is activated with the flag SoftQCD:centralDiffractive $=o n$, and corresponds to the process-number 106. In the event record, the outgoing-protons information is written in 


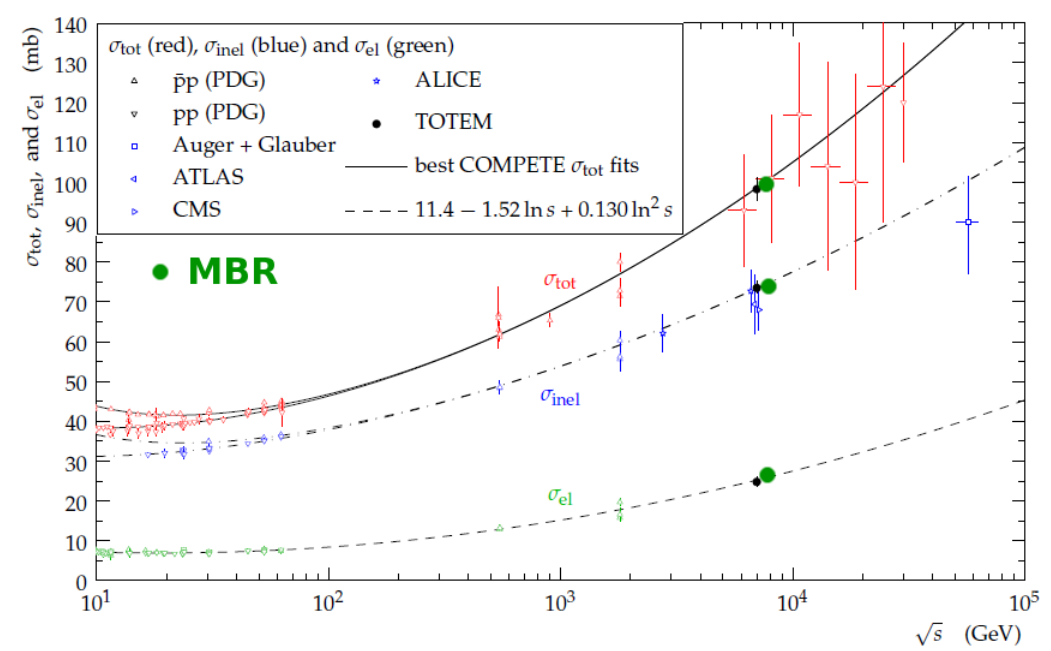

Figure 1: The total, total-inelastic, and elastic $p p$ cross sections at $\sqrt{s}=7 \mathrm{TeV}$ measured by TOTEM[9], shown with best COMPETE fits. The predictions of MBR were added as filled squares.
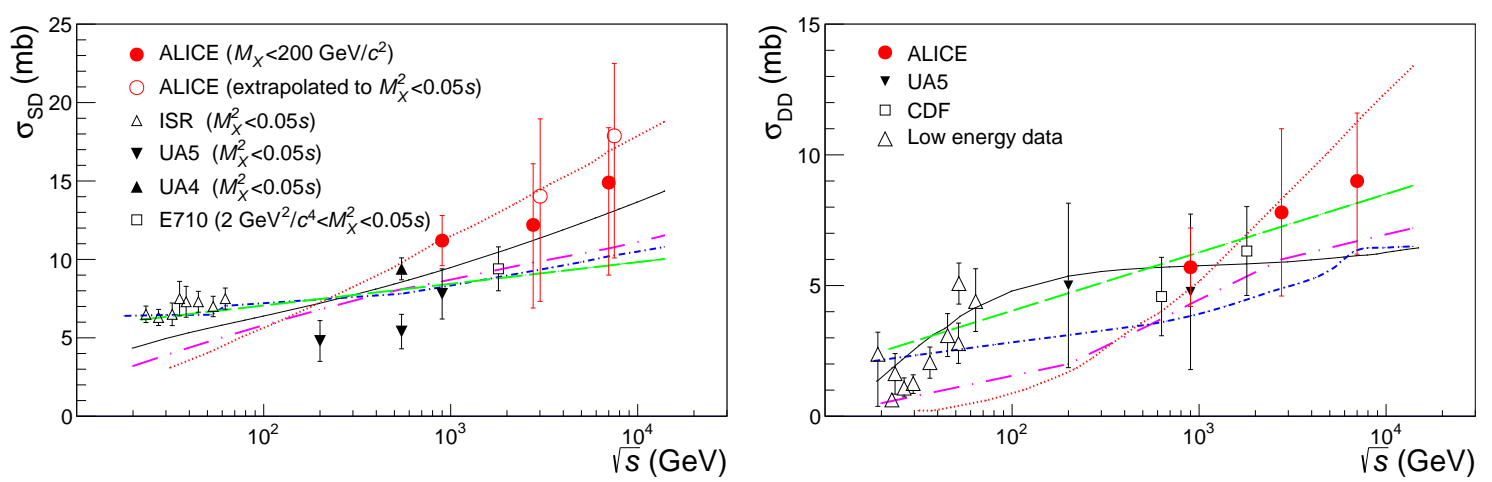

Figure 2: The single-diffractive (left) and double-diffractive (right) cross sections at $\sqrt{s}=0.9,2.76$ and 7 $\mathrm{TeV}$ measured by ALICE[10], compared to predictions of theoretical models. The predictions of MBR are shown as green full curves.

rows 3 and 4, and the centrally-dissociated hadronic system occupies row 5. At the current stage, the hadronization of the dissociated hadronic system(s) is performed using the default PYTHIA 8 machinery. A further development, to be available soon, includes a tune of PYTHIA8 hadronization parameters to reproduce the phenomenological MBR hadronization model[11], based on pre-LHC and pre-Tevatron low-energy data.

\section{Summary}

We presented the MBR (Minimum Bias Rockefeller) Monte Carlo simulation, which has been tested using CDF data, and briefly discussed its implementation in the PYTHIA 8 generator. The double-Pomeron-exchange process is included in PYTHIA8 for the first time. The simulation is designed to apply to all Minimum Bias processes in the LHC energy range. 


\section{References}

[1] R. Ciesielski, K. Goulianos, MBR Monte Carlo Simulation in PYTHIA8, arXiv:1205.1446.

[2] K. Goulianos, Hadronic diffraction: where do we stand?, hep-ph/0407035;

K. Goulianos, Diffraction in QCD, arXiv:hep-ph/020314.

[3] T. Sjöstrand, S. Mrenna and P. Skands, JHEP05 (2006) 026, Comput. Phys. Comm. 178 (2008) 852, arXiv:hep-ph/0603175,arXiv:0710.3820.

[4] R. J. M. Covolan, K. Goulianos, J. Montanha, Phys. Lett. B 389, 176 (1996).

[5] K. Goulianos, Diffraction, Saturation and pp Cross Sections at the LHC, arXiv:1105.4916.

[6] K. Goulianos, Phys. Lett. B358, 379 (1995).

[7] T. Affolder et al. (CDF Collab.), Double Diffraction Dissociation at the Fermilab Tevatron Collider, Phys. Rev. Lett. 87, 141802 (2001). e

[8] D. Acosta et al. (CDF Collab.), Inclusive double Pomeron exchange at the Fermilab Tevatron $\bar{p} p$ collider, Phys. Rev. Lett. 93, 141601 (2004).

[9] G. Antchev et al. (TOTEM Collab.), First measurement of the total proton-proton cross section at the LHC energy of $\sqrt{s}=7$ TeV, Eur. Phys. Lett. 96 (2011) 21002, arXiv:1110.1395.

[10] B. Abelev et al. (ALICE Collab.), Measurement of inelastic, single- and double-diffraction cross sections in proton-proton collisions at the LHC with ALICE, arXiv:1208.4968, submitted to Eur. Phys. J. C.

[11] K. Goulianos, Phys. Lett. B193, 151 (1987). 